\title{
Molecular cloning and expression analysis of mevalonate pyrophosphate decarboxylase in Antrodia cinnamomea
}

\author{
Jing Li ${ }^{1,3}$, Xiong-Jie Lin², En-Si Shao ${ }^{1,3}$ and Zhan-Xi Lin ${ }^{1,3}$ \\ ${ }^{1}$ Fujian Agriculture and Forestry University, Fuzhou 350002, China \\ 2Fruit Research Institute, Fujian Academy of Agriculture Sciences, Fuzhou 350013, China \\ ${ }^{3}$ China National Engineering Research Center of JUNCAO Technology, Fuzhou 350002, China
}

\begin{abstract}
Mevalonate pyrophosphate decarboxylase (MVD) plays important roles in triterpenoid biosynthesis via the mevalonate pathway. A novel MVD gene was isolated and identified in Antrodia cinnamomea (Ac-mvd). The fulllength cDNA contained an open reading frame with a length of 1,209 bp and encoded a 402-amino acid polypeptide with a molecular mass of $43.3 \mathrm{kDa}$ and a theoretical $\mathrm{pI}$ of 8.23 . As incubation time was prolonged for 28 days, the triterpenoid content in the mycelium gradually increased and reached $39.192 \pm 2.025 \mathrm{mg} / \mathrm{g}$; the triterpenoid content of the fruiting body grew in the hay of Cinnamomum kanehirae (ACFB-CK), was $49.391 \pm 2.675 \mathrm{mg} / \mathrm{g}$, which was significantly different from other samples $(P<0.05)$. qRT-PCR revealed that the highest expression levels of Ac-mvd in the mycelium of Antrodia cinnamomea were detected on the 7th day. The expression levels gradually decreased as culture time was extended from 14 days to 42 days.
\end{abstract}

\section{Introduction}

Antrodia cinnamomea (A. cinnamomea) is a traditional medicinal fungus in Taiwan and in the surrounding areas in Asia; this fungus inhabits the empty brown rot cavity of Cinnamomum kanehirae [1]. A. cinnamomea is expensive because of several characteristics, such as high medicinal value, scarcity in nature, and difficulty in artificial cultivation. The fruiting body and mycelium extracts of $A$. cinnamomea have been used as a medicine to improve the activity of organisms in studies involving cell lines and animal models in vivo and in vitro; $A$. cinnamomea extracts provide anti-oxidative [2], anticancer, anti-hepatitic [3], and anti-inflammatory properties [4]. Previous studies showed that the main active ingredients of $A$. cinnamomea are antroquinonol [5], succinic acid, maleic acid [6], benzenoids [7], triterpenoids [8], and polysaccharides [9]. Among these components, triterpenoid constitutes the largest group of phytochemicals in all living organisms [10]; as such, studies have mainly focused on this group. Most triterpenoids are isolated from plants and utilized as commercial flavorings and medicines. Martin et al. [11] devised an alternative method to produce triterpenoids by using Escherichia coli.

Previous studies on plants and fungi confirmed that triterpenoids are synthesized via the mevalonate (MVA) pathway [12]. In 2014, the triterpenoid biosynthesis pathway in $A$. cinnamomea was validated through genomic and transcriptome analyses; this pathway is similar to the MVA pathway in Ganoderma lucidum [13] Several enzymes, such as squalene synthase (SQS) [14], HMG-CoA reductase (HMGR) [15], mevalonate pyrophosphate decarboxylase (MVD) [16], and farnesyldiphosphate synthase (FPS) [17], are implicated in the MVA pathway. A previous study showed that the synthesis of triterpenoids in A. cinnamomea is complex. MVD (EC 4.1.1.33) catalyzes the synthesis of mevalonate-5PP to form isopentenyl-PP, which accumulates to generate monoterpene [18]. Monoterpene is further converted by coenzyme $\mathrm{Q}(\mathrm{CoQ})$ to antroquinonol and ubiquinone; furthermore, SQS catalyzes the conversion of isopentenyl-PP to lanosterol. Lanosterol is an important substrate in the synthesis of ergot sterane and lanosterol triterpenoids by synthesizing cytochrome P450 [13]. The MVD activity has been quantified in animals [19], plants [20], and yeast. [21] MVD can be used as a potential target of several diseases, such as cancer and restenosis [22].

Few studies have focused on the characterization and expression of MVD in $A$. cinnamomea $(A c-m v d)$. In this study, $A c-m v d$ was cloned and characterized. This study aimed to investigate the expression profiles of $A c-m v d$ and the triterpenoid contents in various developmental stages of $A$. cinnamomea mycelium. The results can provide a basis of further studies on the gene function of the triterpenoid biosynthesis in A. cinnamomea and on the development of efficient fermentation processes involving triterpenoids. 


\section{Materials and methods}

\subsection{Strain and culture conditions}

The A. cinnamomea AC001 (GenBank Accession No.: KM925002) was provided by Taiwan Shen-nong Fungal Co., Ltd. The fruiting body of $A$. cinnamomea grew in the hay of $C$. kanehirae (ACFB-CK). The fruiting body that grew in a corn cob (ACFB-CC) was stored in China National Engineering Research Center of JUNCAO Technology. For the shake-flask fermentation with GPM (2.5\% glucose, $0.3 \%$ yeast extract, $0.5 \%$ peptone, $0.3 \%$ maltose, $0.1 \% \mathrm{KH}_{2} \mathrm{PO}_{4}, 0.1 \% \mathrm{MgSO}_{4} \cdot 7 \mathrm{H}_{2} \mathrm{O}, 0.1 \%$ Vitamin $\left.\mathrm{B}_{1}\right), 100 \mathrm{~mL}$ of the medium was placed in a 250 $\mathrm{mL}$ flask and then inoculated with $3 \%$ second-stage precultured broth at $28{ }^{\circ} \mathrm{C}$.

\subsection{Nucleic acid extraction and cDNA synthesis}

The mycelia of $A$. cinnamomea in GMP were harvested after 15 days, frozen in liquid nitrogen, and ground into powder in a mortar. Genomic DNA was extracted by using cetyltrimethyl ammonium bromide (CTAB) method previously described [23]. Total RNA was extracted from different $A$. cinnamomea mycelia and fruiting bodies by using TRIzol reagent (Invitrogen, California, USA). The quality and concentration of the samples were examined using NanoDrop 2000 (Thermo, New York, USA) and $1 \%$ agarose gel electrophoresis. cDNA was synthesized using a first-strand cDNA synthesis kit (TOYOBO, Tokyo, Japan). Each $10 \mu \mathrm{L}$ of reaction mixture contained $500 \mathrm{ng}$ of RNA, $2 \mu \mathrm{L}$ of $4 \times$ DNase Master Mix, and RNase free water at $37{ }^{\circ} \mathrm{C}$ for $5 \mathrm{~min}$; afterward, $2 \mu \mathrm{L}$ of $5 \times$ RT Master Mix was mixed, and the appropriate buffer was added. The reaction mixture was incubated at $37{ }^{\circ} \mathrm{C}$ for $15 \mathrm{~min}$, at $50{ }^{\circ} \mathrm{C}$ for $5 \mathrm{~min}$, and at $98{ }^{\circ} \mathrm{C}$ for $5 \mathrm{~min}$; then, the reaction mixture was subsequently stored at $4{ }^{\circ} \mathrm{C}$.

\subsection{Cloning of the specific fragment of Ac-mvd}

On the basis of the conserved domains in the amino acid sequences of Ganoderma. lucidum, Coriolus versicolor, and Pleurotus ostreatus, we synthesized two degenerate oligonucleotide primers, namely, mvd-1 and mvd-2 (Table 1), for the Ac-mvd amplification of $A$. cinnamomea mycelial cDNA. The PCR amplification conditions were as follows: $95{ }^{\circ} \mathrm{C}$ for $5 \mathrm{~min}$, followed by 32 cycles of denaturation at $94{ }^{\circ} \mathrm{C}$ for $30 \mathrm{~s}$, annealing at $56.5^{\circ} \mathrm{C}$ for 40 $\mathrm{s}$, and extension at $72{ }^{\circ} \mathrm{C}$ for $7 \mathrm{~min}$. The amplification products were transformed into a vector $\mathrm{pMD}^{\mathrm{TM}} 18-\mathrm{T}$ (TaKaRa, Tokyo, Japan) and sequenced by Shanghai Biosune Biotechnology Co., Ltd.

\subsection{Cloning of the full-length Ac-mvd through RACE}

The sequence was used to design the primers for cloning the full-length sequence through the rapid amplification of cDNA ends (RACE).
Table 1. Oligonucleotide primers used in the experiment

\begin{tabular}{cl}
\hline Primer & $5^{\prime}-3^{\prime}$ Sequence \\
\hline mvd-1 & CGTGAACATCGCCTGCATNAARTAYTGGG \\
mvd-2 & CCCTGGACACGTCGTTCADRTARAADAT (R) \\
& \\
mvd-3 & CGACGCCATTCAACCATAG \\
mvd-4 & CCTTCTGGAATGACGGATC \\
mvd-5 & GTTTCCGACGACAAGAAGGG (F) \\
mvd-6 & TCCAGCACCGTATCGCACAT (R) \\
mvd-7 & GCCCTCATTTCTTCATCC (F) \\
mvd-8 & ATACACTCTAGCTCGCCATAA (R) \\
mvd-9 & ACCCTTGACCAAGATCACTTTC (F) \\
mvd-10 & GATATCTTCCTCGACGCCATTC (R) \\
\hline
\end{tabular}

Table 1 shows the PCR amplification conditions using the 5' RACE protocol with Rapid Amplification of cDNA Ends (Invitrogen, California, USA) and 2 specific primers (mvd-3, and mvd-4). The mvd-4 primer was used for the self-formed adaptor (SEFA-PCR) [24], and mvd-4 was used for the nested PCR. The products were transformed into a $\mathrm{pMD}^{\mathrm{TM}} 18-\mathrm{T}$ vector and sequenced.

The 3' RACE sequences were amplified using the 3 'full RACE core set with primescript RTase kit and LA Taq with GC buffer (Takara, Tokyo, Japan). Two specific primers (mvd-5 and mvd-6; Table 1) were designed on the basis of the fragment sequence in accordance with the manufacturer's instructions. PCR amplification was carried out as follows: $94{ }^{\circ} \mathrm{C}$ for $2 \mathrm{~min}, 30$ cycles at $98{ }^{\circ} \mathrm{C}$ for $10 \mathrm{~s}, 55^{\circ} \mathrm{C}$ for $30 \mathrm{~s}, 72{ }^{\circ} \mathrm{C}$ for $2 \mathrm{~min}$, and extension at $75{ }^{\circ} \mathrm{C}$ for $5 \mathrm{~min}$. The product was purified, cloned into $\mathrm{pMD}^{\mathrm{TM}} 18-\mathrm{T}$, and sequenced.

The $3^{\prime}$ and 5' RACE and fragment products of $A$. cinnamomea mycelia were aligned and assembled. The full-length sequence of $A c-m v d$ was amplified using specific primers mvd-7 and mvd-8 (Table 1) with gDNA and cDNA as template. The PCR amplification procedure was carried out as follows: $94{ }^{\circ} \mathrm{C}$ for $5 \mathrm{~min}$, followed by 32 cycles of denaturation at $94{ }^{\circ} \mathrm{C}$ for $30 \mathrm{~s}$, annealing at $52.8{ }^{\circ} \mathrm{C}$ for $30 \mathrm{~s}$, and elongation at $72{ }^{\circ} \mathrm{C}$ for $40 \mathrm{~s}$, with extension at $72{ }^{\circ} \mathrm{C}$ for $7 \mathrm{~min}$. The product was purified, cloned into $\mathrm{pMD}^{\mathrm{TM}} 18-\mathrm{T}$, and sequenced.

\subsection{Bioinformatics analysis}

The full-length sequence of $A c-m v d$ was identified, and homologs were analyzed in accordance with the BLASTP algorithm of NCBI (http://blast.ncbi.nlm.nih.gov/Blast.cgi). Multiple alignments were carried out using Clustal X 2.0 and the MEGA 5.1 [25]. Data were analyzed using the Maximum Likelihood Method (ML) [26], and the bootstrap strength was supported by 1000 replicates for both in ML analysis. The complete amino acid sequences of each fungus were used to construct the phylogenic tree. The protein sequences were analyzed using the bioinformatics tools ExPASy, SignalP4.1 Sever, and TMHMM Server2.0. The secondary and tertiary structures of the $A c-m v d$ protein were predicted using the SWISS-MODEL Protein Modeling Server (http://swissmodel.expasy.org/) and InterPro Protein (http://www.ebi.ac.uk/interpro/). Further analysis was carried out using PyMOL. 


\subsection{Expression analysis of Ac-mvd through quantitative real-time PCR (qRT-PCR) in different development stages}

The mycelia of A. cinnamomea were harvested after the fungus was cultured in GPM for 7, 14, 21, 28, 35, and 42 days. Total RNA was isolated from each stage, and the synthesized cDNA was used as a template for qRT-PCR amplification with two primers, namely, mvd-9 and mvd10 (Table 1). qRT-PCR was performed using CFX Connect (Bio-rad, California, USA) in $20 \mu \mathrm{L}$ of reaction mixture containing $10 \mu \mathrm{L}$ of SYBR Premix Ex Taq ${ }^{\mathrm{TM}}$ (TaKaRa, Tokyo, Japan), $1 \mu \mathrm{L}$ of sample cDNA, $2 \mu \mathrm{L}$ of primers, and $7 \mu \mathrm{L}$ of sterile double-distilled water. The cDNA of ACFB-CK was used as a positive control. Double-distilled water instead of first-strand cDNA was used as a negative control.

\subsection{Extraction and analysis of triterpenoids in the mycelium and fruiting body of $A$. cinnamomea}

The total triterpenoids of $A$. cinnamomea mycelium were extracted through ultrasonic treatment (Kun Shan Ultrasonic Instruments Co., Ltd., Jiangsu, China). Dried mycelia and fruiting body powder $(0.5 \mathrm{~g})$ were mixed with $40 \mathrm{~mL}$ of $80 \%$ ethanol and ultrasonic wave at $70{ }^{\circ} \mathrm{C}$ for $2 \mathrm{~h}$. The slurry was centrifuged at $7000 \mathrm{rpm}$ for 20 min to collect the supernatant, and the supernatant volume was diluted with $80 \%$ ethanol to $50 \mathrm{~mL}$.

Each sample $(0.5 \mathrm{~mL})$ was placed in a scale tube and dried at $50{ }^{\circ} \mathrm{C}$. Afterward, $0.3 \mathrm{~mL}$ of $5 \%$ vanillic-acetic acid and $1 \mathrm{~mL}$ of perchloric acid were added to the tubes, and the reaction was mixed at $60{ }^{\circ} \mathrm{C}$ for $20 \mathrm{~min}$. Acetic acid $(10 \mathrm{~mL})$ was added to the tubes, and the reaction was mixed after cooling [27]. The total triterpenoid content was determined by measuring the absorbance at $548 \mathrm{~nm}$ with a spectrophotometer (Biochrom, Cambridge, England), and oleanolic acid was used as the standard. The concentration of triperpenoids was calculated using Eq. (1):

$$
\text { Total triperpendiod }(\mathrm{mg} / \mathrm{g})=\frac{\text { triperpendiodsquality insample }}{\frac{\text { weight of sample }}{\text { cons } \tan t \text { volume }} \times \text { volumeof sample }}
$$

\subsection{Statistical analysis}

Data were expressed as the average of three independent sample measurements. Error bars indicated the standard deviations from the triplicates. The difference between contrasting treatments was considered significant at $P<$ 0.05. Ac-mvd levels were determined according to a standard equation curve $\left(\mathrm{Y}=-3.1854 \mathrm{X}+43.264, \mathrm{R}^{2}=\right.$ 0.996 , Log Starting quantity (Log SQ) is $\mathrm{X}, \mathrm{Cq}$ Value is Y) [28]. The gene copies were calculated using Eq. (2). Gene copies $($ copies $/ \mu L)=6.02 \times 10^{23} \square(500 \mathrm{ng} / \mu L) / c D N A$ Length $\square 660$

\section{Results}

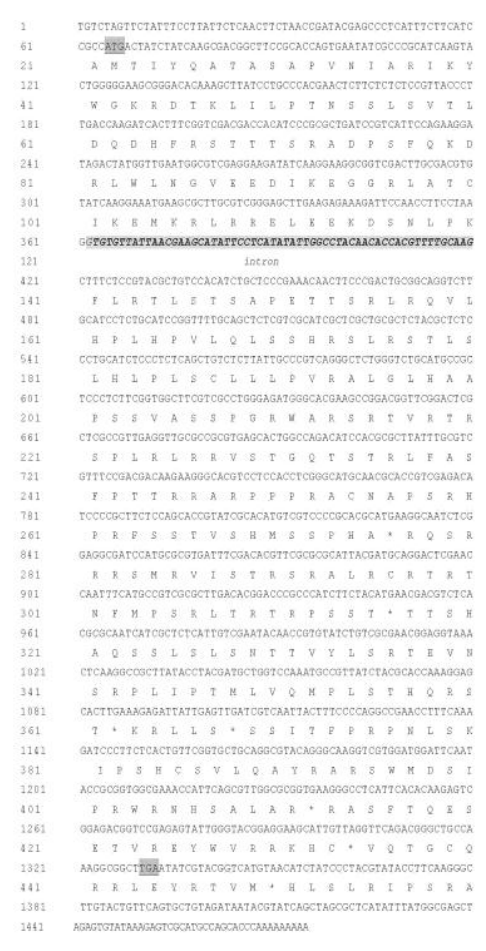

Figure 1. Promoter region, genomic DNA, and predicted amino acid sequence of $A c-m v d$. The intron of mvd was shaded in bold italics and light gray.

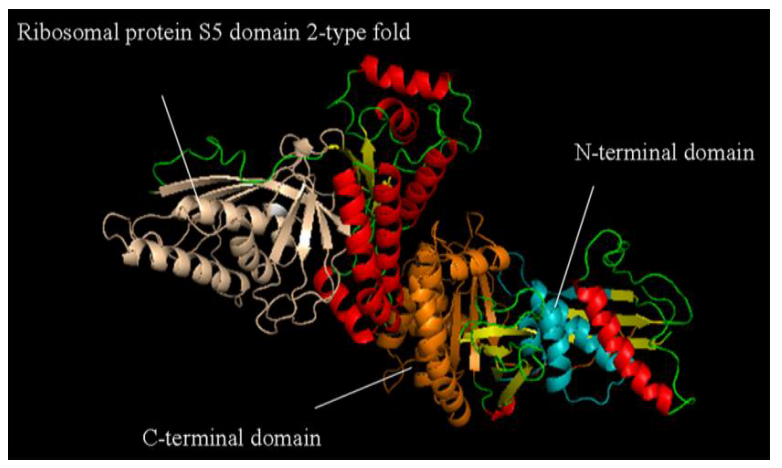

Figure 2. 3-D structural model of the amino acid sequences of Ac-mvd. The structural model of $A c-m v d$ was created on the basis of Gl-mvd (3f0n.1.A). The pI of MVD is 8.23, and no signal peptide and transmembrane domain are detected. The hydrophobic and hydrophilic regions are shown in the protein conformation.

\subsection{Cloning and Characterization of Ac-mvd}

A novel full-length cDNA clone of $A c-m v d$ was generated from A. cinnamomea. The $809 \mathrm{bp}$ fragment of $A c-m v d$ was obtained from cDNA via PCR by using two degenerate primers, namely, mvd-1 and mvd-2. The product was homologous to $m v d$ in G. lucidum, Trametes cinnabarina, Trametes versicolor, Ceriposiospis subvermispora, and Dichomitus squalens. The $3^{\prime}$ and 5' RACE products were amplified depending on the obtained specific $809 \mathrm{bp}$ fragment. The product length of 3' RACE was approximately $690 \mathrm{bp}$; the product length 
of 5' RACE was approximately $197 \mathrm{bp}$. The full-length $A c-m v d$ was obtained using the mvd-7 and mvd-8primers with cDNA and gDNA as the respective templates. The 1268 bp full-length gDNA was composed of a 59 bp intron (Fig. 1). The full-length cDNA encoded a 402amino acid polypeptide with a theoretical molecular mass of $43.3 \mathrm{kDa}$. The polypeptide contained the distinctive domain of the ribosomal protein S5 domain; the polypeptide was also similar to the MVD of G. lucidum (Fig. 2). The nucleotide sequence of cDNA (Ac-mvd) was deposited in GenBank with the accession number KR364808.

\subsection{Phylogenetic analysis}

A phylogenetic tree was generated on the basis of the deduced amino acid sequences to determine the evolutionary relationship between $A c-m v d$ and other fungi; the BLASTX search showed that the Ac-mvd fragment was highly homologous to the other fungi (Fig. 3 ). Most of the predicted homologous proteins of Ac-mvd in D. squalens ( $88 \%$ identity), G. lucidum ( $86 \%$ identity), T. versicolor ( $85 \%$ identity), Rhizoctonia solani $(79 \%$ identity), and Cryptococcus gattii (70\% identity) were selected and subjected to phylogenetic analysis. $m v d$ is generally found in many fungi, whereas the homologous $m v d$ sequences were divided into the following groups: basidiomycetes (G. lucidum and T. versicolor) and yeast (Schizosaccharomyces cryophilus and C. gattii).

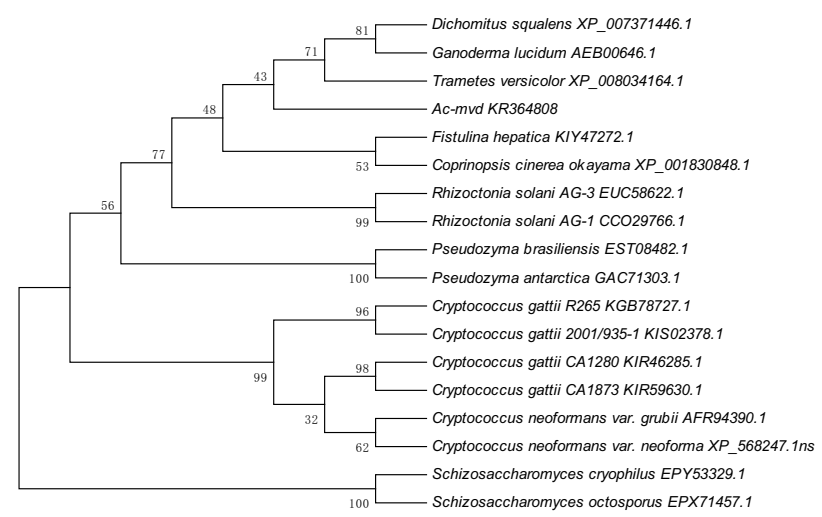

Figure 3. Phylogenetic tree showing the relationship of $A c-m v d$ with other proteins from various fungi.

\subsection{Ac-mvd expression and triterpenoid concentration in the mycelium of $A$. cinnamomea in different development stages}

The cDNA was synthesized as a template to determine the expression levels of Ac-mvd in different stages through absolute qRT-PCR. The gene expression levels of $A c-m v d$ were calculated from 7 days to 42 days; the results revealed that the expression levels decreased sharply from $47.3 \times 10^{5}$ copies $/ \mu \mathrm{L}$ to $35.5 \times 10^{4}$ copies $/ \mu \mathrm{L}$. ACFB-CK and ACFB-CC contained $49.8 \times$ $10^{4}$ and $21.1 \times 10^{4}$ copies $/ \mu \mathrm{L} A c-m v d$, respectively (Fig. 4).

The submerged culture of $A$. cinnamomea mycelia and fruiting bodies was dried and used to extract and determine the triterpenoid content of the fungus. The triterpenoid contents in $A$. cinnamomea mycelia were $15.170 \pm 1.400,17.574 \pm 2.015,26.400 \pm 2.259,39.192 \pm$ $2.025,28.907 \pm 0.619$, and $30.074 \pm 0.684 \mathrm{mg} / \mathrm{g}$ corresponding to the culture after 7, 14, 21, 28, 35, and 42 days (Fig. 5), respectively. As culture time was prolonged, the triterpenoid content of $A$. cinnamomea mycelia increased from 7 days to 28 days and then decreased from 35 days to 42 days. The triterpenoid contents of ACFB-CK and ACFB-CC were $49.391 \pm$ 2.675 and $11.530 \pm 0.733 \mathrm{mg} / \mathrm{g}$, respectively, which were significantly higher than the triterpenoid contents in all of the development stages of the mycelia $(P<0.05)$ of other samples in ACFB-CK. By contrast, the lowest triterpenoid content was determined in ACFB-CC among the mycelium samples (Fig. 5).

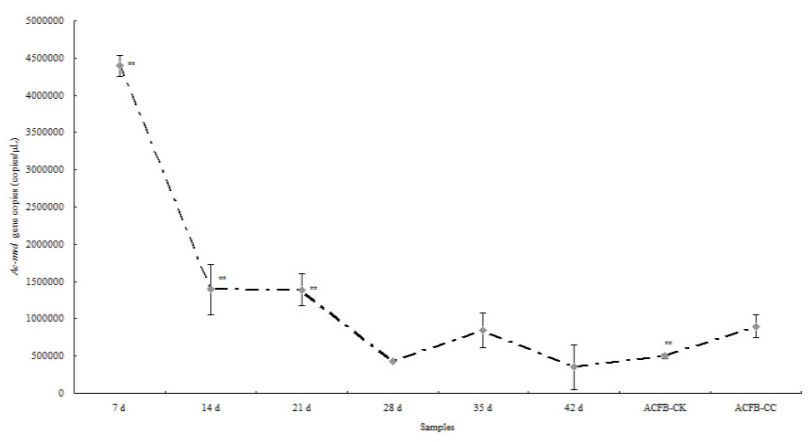

Figure 4. A. cinnamomea mycelia $A c-m v d$ gene copy numbers in different developmental stages $(* * P<0.05)$

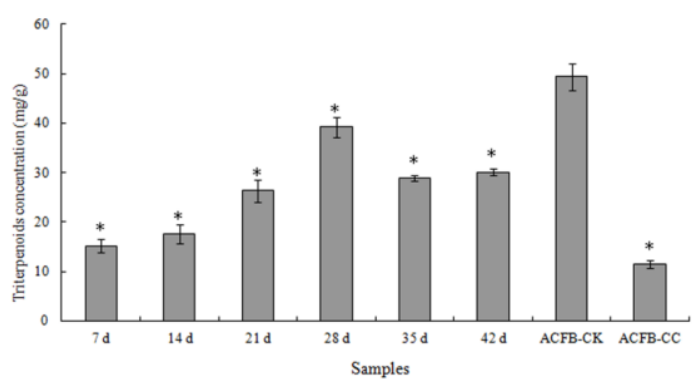

Figure 5. Triterpenoid contents of A. cinnamomea mycelia in a submerged medium in different stages; ACFB-CK was used as a control $(* \mathrm{P}<0.05)$

\section{Discussion}

Previous studies demonstrated that the triterpenoid biosynthesis of $A$. cinnamomea is significantly affected by several environmental factors, such as light, elicitor, substrate, $\mathrm{pH}$ value, and developmental stages [29-31]. The gene expression levels of $A c-m v d$ were the highest in the mycelia of $A$. cinnamomea on the 7 th day of the submerged culture. The highest triterpenoid content was detected in ACFB-CK; conversely, the triterpenoid content of A. cinnamomea mycelia was slightly lower than that of ACFB-CK on the 28th day in the submerged culture. The lowest gene expression levels and triterpenoid content were detected in ACFB-CC. However, the absolute qRT-PCR results showed that the 
Ac-mvd expression level was not linearly correlated with the triterpenoid content.

Triterpenoids exhibit important medicinal properties in vivo and in vitro. In a previous study, the overexpression of $m v d$ is positively correlated with gene expression and triterpenoid content in G. Lucidum[16]. In the triterpenoid biosynthesis pathway of $A$. cinnamomea, MVD catalyzes the synthesis of mevalonate-5PP to form isopentenyl-PP, which is the substrate of zeatin and monoterpenoid biosynthesis. The main Kyoto Encyclopedia of Genes and Genomes (KEGG) mapping of the MVA pathway in A. cinnamomea shows that Ac$m v d$ is a differentially expressed gene in mycelia and fruiting bodies. To our knowledge, the Ac-mvd gene expression was significantly different between ACFB-CC and ACFB-CK in the mycelia cultured for 7 days. The triterpenoid content was decreased as culture time was prolonged. However, $A c-m v d$ expression was not directly proportional to $A c-m v d$ and triterpenoid, and this finding is distinct from that observed in G. lucidum. The MVA pathway is mainly involved in triterpenoid synthesis; the MVA pathway also provides a comprehensive collection of enzymes in the production of major metabolites. Metabolites can block polyisoprenoid and sterol biosynthesis in the MVA pathway [32]. Using 6fluoromevalonic acid in tissue extracts, researchers found that MVD is the target of a downstream metabolite that blocks sterol production[33]. In our study, the triterpenoid content accumulated in the mycelium and Ac-mvd gene expression was reduced when the incubation time was extended.

In conclusion, a novel gene was cloned and characterized from $A$. cinnamomea. The Ac-mvd gene expression patterns in different stages of $A$. cinnamomea mycelia and fruiting bodies were also different. The triterpenoid content may be related to the gene expression level and accumulation time of the synthesis pathway. The successful cloning and expression analysis of $A c$ $m v d$ provide a framework for further studies on the function and triterpenoid synthesis in A. cinnamomea.

\section{Acknowledgments}

This work was supported by the Collaborative Innovation Center for Juncao Ecology Industry under Grant Fujian 2011 Program, k80nd8002 and the China National Engineering Research Center of JUNCAO Technology under Grant 2011FU125X12.

\section{References}

1. T.T. Chang, W.N. Chou. Mycological Research, 1995, 99(6).

2. C.H. Shu, M.Y. Lung. Journal of the Chinese Institute of Chemical Engineers, 2008, 39(1).

3. Y.C. Ho, M.T. Lin, K.J. Duan, Y.S. Chen. Journal of the Chinese Institute of Chemical Engineers, 2008, 39(5).

4. C.L. Wen, C.C. Chang, S.S. Huang, C.L. Kuo, S.L. Hsu, J.S. Deng, G.J. Huang. Journal of ethnopharmacology, 2011, 137(1).
5. V.B. Kumar, T.C. Yuan, J.W. Liou, C.J. Yang, P.J. Sung, C.F. Weng. Mutation Research/Fundamental and Molecular Mechanisms of Mutagenesis, 2011, 707(1).

6. W.L. Lin, Y.J. Lee, S.M. Wang, P.Y. Huang, T.H. Tseng. European journal of pharmacology, 2012, 680(1).

7. H.M. Lien, H.W. Lin, Y.J. Wang, L.C. Chen, D.Y. Yang, Y.Y. Lai, Y.S. Ho. Evidence-Based Complementary and Alternative Medicine, 2011, 2011.

8. T.W. Ma, Y. Lai, F.C. Yang. Bioprocess Biosyst Eng, 2014.

9. M.C. Tsai, T.Y. Song, P.H. Shih, G.C. Yen. Food chemistry, 2007, 104(3).

10. A. Bishayee, S. Ahmed, N. Brankov, M. Perloff. Frontiers in bioscience: a journal and virtual library, 2011, 16.

11. V.J. Martin, D.J. Pitera, S.T. Withers, J.D. Newman, J.D. Keasling. Nature biotechnology, 2003, 21(7).

12. T. Goodwin. Biochemical Journal, 1971, 123(3).

13. M.Y.J. Lu, W.L. Fan, W.F. Wang, T.C. Chen, Y.C. Tang, F.H. Chu, T.T. Chang, S.Y. Wang, M.Y. Li, Y.H. Chen. Proceedings of the National Academy of Sciences, 2014, 111(44).

14. J.S. Zhou, S.L. Ji, M.F. Ren, Y.L. He, X.R. Jing, J.W. Xu. Biochemical Engineering Journal, 2014, 90.

15. C.H. Shang, F. Zhu, N. Li, X. Ou-Yang, L. Shi, M.W. Zhao, Y.X. Li. Bioscience, Biotechnology, and Biochemistry, 2008, 72(5).

16. L. Shi, L. Qin, Y. Xu, A. Ren, X. Fang, D. Mu, Q. Tan, M. Zhao. Molecular biology reports, 2012, 39(5).

17. Y.X. Ding, X. Ou-Yang, C.H. Shang, A. Ren, L. Shi, Y.X. Li, M.W. Zhao. Bioscience, Biotechnology, and Biochemistry, 2008, 72(6).

18. K. Bloch, S. Chaykin, A. Phillips, A. De Waard. Journal of Biological Chemistry, 1959, 234(10).

19. M. Alvear, A.M. Jabalquinto, J. Eyzaguirre, E. Cardemil. Biochemistry, 1982, 21(19).

20. D. Skilleter, R. Kekwick. Biochem. J, 1971, 124.

21. J.K. Bartz, D. Söll. Biochimie, 1972, 54(1).

22. M.J. Toth, L. Huwyler. Journal of Biological Chemistry, 1996, 271(14).

23. M.A. Saghai-Maroof, K.M. Soliman, R.A. Jorgensen, R.W. Allard. Proceedings of the National Academy of Sciences of the United States of America, 1984, 81(24).

24. S. Wang, J. He, Z. Cui, S. Li. Appl Environ Microbiol, 2007, 73(15).

25. K. Tamura, D. Peterson, N. Peterson, G. Stecher, M. Nei, S. Kumar. Molecular biology and evolution, 2011, 28(10).

26. A. Jagerschmidt, N. Guillaume, B.P. Roques, F. Noble. Molecular pharmacology, 1998, 53(5).

27. Z.M. Lu, W.Y. Tao, H.Y. Xu, J. Lim, X.M. Zhang, L.P. Wang, J.H. Chen, Z.H. Xu. Food chemistry, 2011, 127(2).

28. P. Cawthon's quantitative real-time. Biotechniques, 2008, 44.

29. F.C. Yang, H.C. Huang, M.J. Yang. Enzyme and Microbial Technology, 2003, 33(4). 
30. C.H. Shu, M.Y. Lung. Journal of the Chinese Institute of Chemical Engineers, 2008, 39(1).

31. C.J. Liu, C.C. Chiang, B.H. Chiang. Biochemical Engineering Journal, 2012, 64(0).
32. H.M. Miziorko. Archives of biochemistry and biophysics, 2011, 505(2).

33. J. Nave, H. d'Orchymont, J. Ducep, F. Piriou, M. Jung. Biochem. J, 1985, 227. 